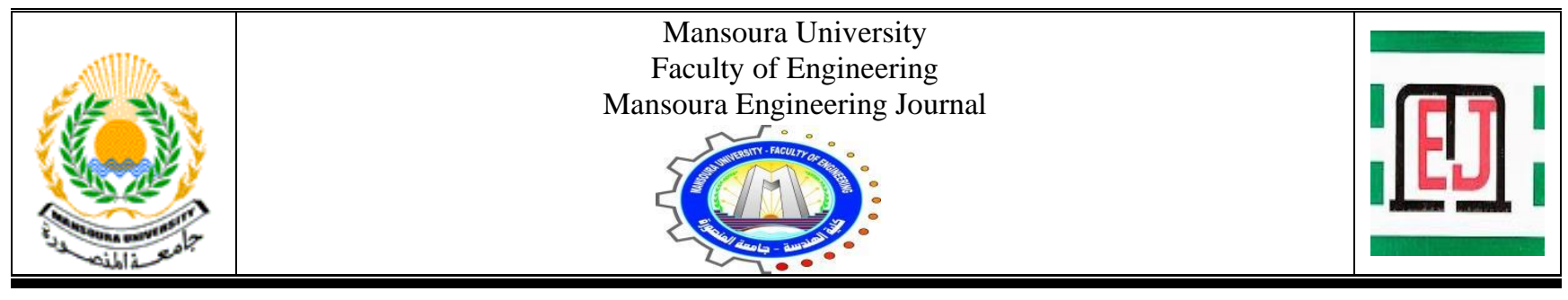

\title{
Performance Evaluation and Enhancement of Medical Imaging Using Plasmonic Gold Nanostars
}

\author{
Kholoud Ramadan*, Hossam El-Din Moustafa and Bedir B.Yousif
}

\begin{tabular}{|l|}
\hline KEYWORDS: \\
Plasmonic \\
nanoparticles, gold \\
nanostars, biomedical \\
imaging, plasmon \\
resonance, \\
nanoparticles dimmers. \\
\\
\end{tabular}

Abstract- This paper gives insights about examinations of plasmonic nanoparticles (NPs) and particularly gold nanostar as sign enhancer and contrast agent for clinical imaging and the optical properties of (NPs) of different volume and morphology and the encompassing medium were stood out from affirm that the gold nanostars are the best shape that can scatter the light, thusly obtaining better and all the clearest clinical imaging. Stars formed gold nanoparticles have a greater surface comparative with its volume and contact surface cross section than spherical and rods shaped nanoparticles, and accordingly act even more firmly as a radiation enhancer. At that point the wavelength of the surface plasmon resonance (SPR) for nanostars is (700 to 900 $\mathrm{nm})$ in the body tissues transparence window range, while it from (500 to 700 nm) with nanospheres and from $(600$ to $800 \mathrm{~nm})$ with nanorods, the laser can impact gold nanostars more profound inside the human body. Mathematical outcomes exhibit that the gold nanostar has more powerful scattering cross section conversely with sphere and rod shaped gold nanoparticles. The largest nanostar displayed better contrast properties for imaging applications, for instance, confocal microscopy and optical coherence tomography.

\section{INTRODUCTION}

$\mathrm{O}$ PTICAL imaging exemplifies one the very pinnacle of fundamental apparatuses in biological studies. Despite the fact that with incredible advances, optical imaging actually experiences hitch like

Received: (26 September, 2021) - Revised: (13 December, 2021) Accepted: (16 December, 2021)

*Corresponding Author: Kholoud Ramadan, Researcher at Electronics and Communications Dept., Faculty of Engineering-Mansoura UniversityMansoura-Egypt (e-mail: kholoudalashmawy@gmail.com).

Hossam El- Din Moustafa, Professor with the Electronics and Communications Dept., Faculty of Engineering- Mansoura UniversityMansoura-Egypt (e-mail: hossammoustafa@ieee.org).

Bedir B.Yousif, Associate Professor with Electrical Engineering Dept., Faculty of Engineering, Kafrelsheikh University- Kafrelsheikh-Egypt- at Electrical Engineering Dept., Faculty of Engineering and Information Technology, Onaizah Colleges, Al-Qassim, KSA. (e-mail: bedir.yousif@gmail.com- bedir.yousif@oc.edu.sa). resolution, sensitivity, speed, and penetration depth. Attributable to the selective optical properties of gold nanoparticles (AuNPs),in another meaning surface plasmon resonance, AuNPs canister is promptly used to improve optical images set up on their absorption, scattering, fluorescence, raman scattering, etc. and so on Here, we incorporate the most current accomplishments and provokes specialist with utilizing AuNPs to propel goal and affectability in medical imaging. Utmost biomedical applications requisite particle plasmon resonance wavelengths in the range from 700 to $1300 \mathrm{~nm}$ that resemble to the tissue transparence window. Altering the geometrical shape of nanoparticles is one of the ways to control the position of the plasmon resonance peak. Plasmon resonant for gold nanoparticles require usual significant attention as a potential technology in optical imaging, laser diagnostics and medication with the interesting property of (PR) ensuing in an upgraded electromagnetic field on the metallic (NPs) surfaces (Kim EY et al. 2015; Kanika 
Khurana and Neena Jaggi (2021); Luca Guerrini et al. 2021). Thus, exceptional consideration is as of now being paid to anisotropic AuNPs with a plasmon resonance which is tunable to the close by infrared (NIR) district inside the transparent range (700 to1300 $\mathrm{nm}$ ) with upgraded photons (light) entrance into biotissue. Remarkable to this property, lower toxicity, simple function and stability, anisotropic AuNPs can be utilized for treatment and diagnostics (Versiani AF et al. 2016; Sousa-Castillo et al. 2016; Bhamidipati et al. 2017). Anisotropic extended gold nanoparticles especially gold nanostars (AuNSts), have become especially fast approaching competitor for applications in biomedicine because of their wide plasmonic resonance peaks and extremely high electric field intensity concentrated at their branches (Duke University (2020); Jana D et al. 2015; Vendrell M et al. 2013; Harmsen S et al. 2015). Exceptionally, at this moment of the presented study outstandingly, most of gold nanostar applications in medical imaging and detecting are restricted to surface enhanced raman scattering (spectroscopy). In any case, due to extraordinary dispersing (scattering) properties, AuNSts assurance to be splendid optical imprints for various imaging applications. For example, the usage of gold nanostar (AuNSt) as contrast agent for (OCT) imaging is valuable for forwarddissipating (scattering) fluids since nanoparticles will increase backward reflected photons, making conceivable static fluid very well imaging, yet furthermore diversion of the fluid speed profile, which is critical for infection diagnostics. Another valuable resource for imaging cell and shockingly nuclear changes is confocal laser scanning microscopy (CLSM) that furnishes that furnishes 3D minuscule images with a bewildering sub-micron spatial resolution. The usage of AuNSts CLSM builds the backscattered signal from the examples for the direct synchronized management of the takeup and localisation of nanoparticles in cells. The nonappearance of conceivably poisonous fluorescent dyes in the examples empowers zeroing in on the impact of the AuNPs on the cells solely, which is generally critical for biomedical applications.

In this paper, we report to provide an in-depth basic understanding of the plasmonic properties of gold nanostars. We have shown how we were able to successfully establish a causal relationship between particle morphology and its measured plasmonic behavior. Then choose the best form that can be used to improve the quality of medical imaging and thus accurately diagnose diseases.

First, we simulate the nanosphere, nanorod, and nanostar using the (COMSOL Multiphysics) program and investigate the properties of each nanoparticle shape and study the effect of some factors such as changing the surrounding medium, nanoparticle size and nanoparticulate dimer on the properties of each morphology. Then we choose the most appropriate morphology to use for this task, which became clear from the results that the nanostar has distinctive properties that can be benefited from and developed for use as a contrast factor to improve medical imaging.

\section{DESIGN AND MOdeling NANOPARTICleS}

In this work initially, the particles of spheres, rods and nanostars were recreated and modulated by using finite element method (FEM) (Hsiangkuo et al. 2012; Zhihua et al. 2015). Utilizing episode light source as found in mathematical equation (1). The model of nanostar and their optical properties will studies using RF module of (COMSOL Multiphysics 5.0).

$$
E=e^{-j k_{0} z} \hat{X}
$$

The simulation was based on numerical solutions of a time- averaged Laplacian, seen in equation (2), employing the widely used finite element.

$$
\nabla \times \frac{1}{\mu_{r}}(\nabla \times E)-k_{0}^{2}\left(\varepsilon_{r}-\frac{j \sigma}{\omega \varepsilon_{0}}\right) E=0
$$

The geometry was based on three concentric areas. A completely absorption spherical layer, typically alluded to as perfect matching layer (PML), the layer appointed the dielectric properties of water, (Hale et al. 1973) and a center space including the different nanostar models allocated the dielectric characteristics of gold from the (Drude-Lorentz) just vaules from Christy and Johnson (Johnson et al. 1972) The relative permeability $\left(\mu_{\mathrm{r}}\right)$ taken to be one in all calculation areas. The angular frequency $\omega$ determined from the input wavelengths values.

The models for nanostars were also developed in COMSOL Multiphysics using at most a spherical core, eccentric conical spikes with hemispherical, oblate, and prolate hemi ellipsoidal tips. The volume and surface areas of these models are defined by equations (3) and (4) with i being the number of spikes.

$$
\begin{aligned}
& \mathrm{V}=\frac{4}{3} \pi a^{3}+\sum_{\mathrm{i}=1}^{\mathrm{n}}\left\{\frac{\mathrm{h}_{\mathrm{i}}}{3} \pi\left(\mathrm{R}_{\mathrm{i}}^{2}+\mathrm{R}_{\mathrm{i}} \mathrm{r}_{\mathrm{i}}+\mathrm{r}_{\mathrm{i}}^{2}\right)+\frac{2}{3} \pi \mathrm{r}_{\mathrm{i}}^{3}-\right. \\
& \left.\frac{\pi}{6}\left(\mathrm{a}-\sqrt{\mathrm{a}^{2}-\mathrm{R}_{\mathrm{i}}^{2}}\right)\left[3 \mathrm{R}_{\mathrm{i}}^{2}+\left(\mathrm{a}-\sqrt{\mathrm{a}^{2}-\mathrm{R}_{\mathrm{i}}^{2}}\right)^{2}\right]\right\}
\end{aligned}
$$

$\mathrm{A}=4 \pi a^{2}-\sum_{i=1}^{n}\left\{2 \pi a\left(a-\sqrt{a^{2}-R_{i}^{2}}\right\}+\sum_{i=1}^{n}\left\{\pi\left(r_{i}+R_{i}+\right.\right.\right.$ $\left.2 \pi r_{i}^{2}\right\}$

Moreover, (a) is the radii of the (sphere core), (R) is the base radii of a (spike), (r) is the radii of the (hemisphere tip of a spike), and (h) is the (distance between $R$ and $r$ ).

From the solution of equation (2), provide plots of the normalized scattered electrical fields. Three dimensional plots of the electrical field norm (equation 5) on the surface of the various NSs along with two-dimension slice plots of the electric fields' norm are presented. 2D slice plots are fundamental to study, at the same time, surface and bulk plasmons modes in these nanoparticles.

$|E|=\sqrt{E_{X}^{2}+E_{Y}^{2}+E_{Z}^{2}}$ 
In parallel, calculate the scattering, absorption and extinction, as defined by equations (6) and (7).

$\sigma_{\mathrm{abs}}=\frac{\mathrm{W}_{\mathrm{abs}}}{\mathrm{P}_{\mathrm{inc}}} \quad \sigma_{\mathrm{sca}}=\frac{\mathrm{W}_{\mathrm{sca}}}{\mathrm{P}_{\mathrm{inc}}}$

$\sigma_{\mathrm{ext}}=\sigma_{\mathrm{abs}}+\sigma_{\mathrm{sca}}$

Absorption and scattering are definite by the energy rates (absorbed and scattered) by the nanostars due to its size, defined in equations (8) and (9) respectively, and divided by the incident irradiance, given by equation (10).

$\mathrm{W}_{\mathrm{abs}}=\frac{1}{2} \iiint_{\mathrm{xyz}}\left[\operatorname{Re}\left[(\sigma \mathrm{E}+\mathrm{j} \omega \mathrm{D}) \cdot \mathrm{E}^{*}\right]+\right.$

jwB). $\left.H^{*}\right] d x$ dy dz (W)

$\mathrm{W}_{\mathrm{sca}}=\oiint\left[\mathrm{E} \times \mathrm{H}^{*}\right]$. nds $(\mathrm{W})$

$\mathrm{P}_{\mathrm{inc}}=\frac{1}{2 \mathrm{Z}_{0}}\left|\mathrm{E}_{0}\right|^{2}$

E0 is the amplitude of the episode electrical fields $(1 \mathrm{~V} / \mathrm{m})$ and $\mathrm{Z} 0$ is the impedance of free space as found in equation (1). Also, given the extinction, we compute the morphology and concentration dependence extinction coefficient by multiplying the extinction with the number of density for nanoparticles as see in equation (11):

$\varepsilon_{\text {dep }}=\sigma_{\text {ext }} \mathrm{n}$

Similarly, the coefficient of absorption is given by equation (12):

$\mathrm{a}=\sigma_{\mathrm{abs}} \mathrm{n}$

$\mathrm{Au}$ and $\mathrm{Ag}$ nanostructures are utilized as plasmonic biosensors for the discovery of explicit proteins in bio-molecules that are applicable for explicit infections (Haes AJ et al. 2002; Ginting et al. 2017). Extinctions bands of such nanostructures relies upon the refractive indices of the medium adjacent them; for the greater refractive index, it goes through a red shift (Anker JN et al. 2008). The capacity of scattering is the example is pivotal for imaging applications, for example, optical coherence tomography (OCT). It is known, that for nanospheres and nanorods the absorption is the main contributor in the extinction cross section spectrum (Jain PK et al. 2006; Xianghua Meng et al. 2016; Swarnapali D. S. Indrasekara et al. 2015; Helena de Puig et al. 2015) that can be an issue for optical medical imaging techniques, while the scattering is the main contributor in nanostars extinction cross section as shown in Figure (1, 2 and 3). Conversely, nanostars possess a significant ability for scattering. The large enhancement of electromagnetic fields at the tips of the star is because of a collection of the enhancement in resonance and the effect of lighting rod correlating at the tips with the larger curvature. A bigger surface charges densities and thus a higher electrical fields Occurs because this curvature. Accordingly, we researched and thought about the optical characteristics of AuNSt of different sizes and different number of spikes as attractive contrast agent, focusing on their scattering abilities and optical properties.

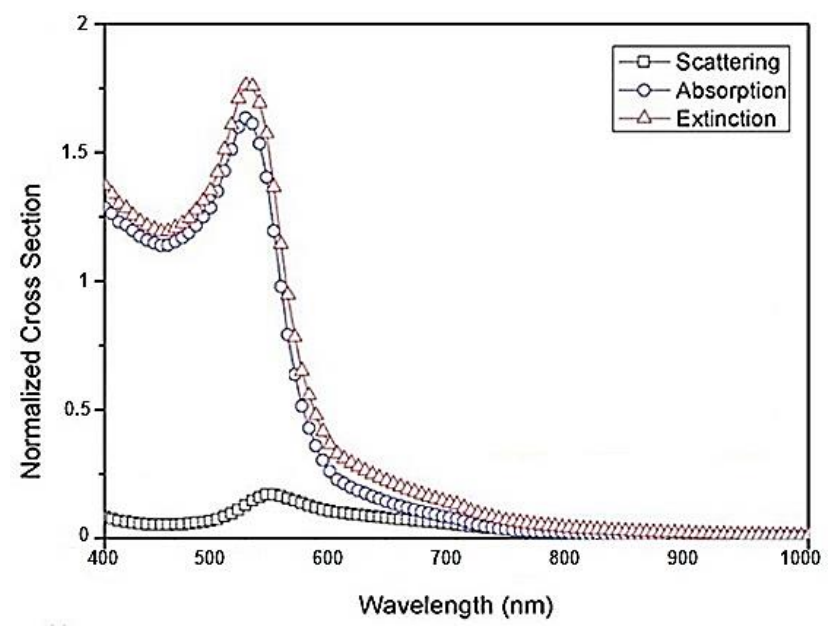

Fig 1: Extinction, absorption and scattering spectrum for gold nanosphere.

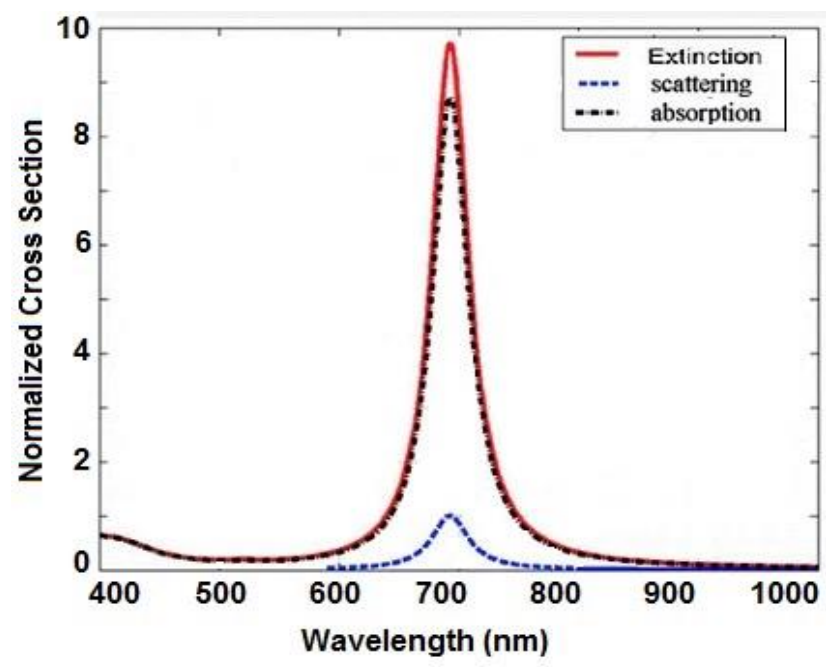

Fig 2: Extinction, absorption and scattering spectrum for gold nanorod.

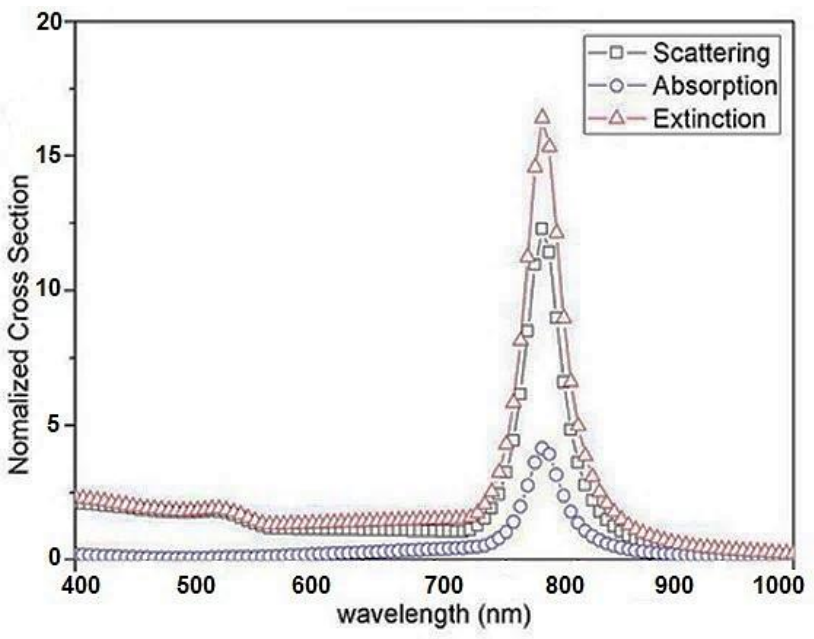

Fig 3: Extinction, absorption and scattering spectrum for gold nanostar. 


\section{ReSUlts}

\section{1- Gold Nanospheres}

The first and the simplest type of nanostructures are sphere gold nanoparticle. When diameters of AuNSps around (30120) $\mathrm{nm}$, the efficient for scatter light increased in the visual range of the spectra, approximately the power of six of nanosphere linear size the intensity of scattered light will occurs. It is a well-known fact, that smaller particle mostly absorbs light, while large ones scatter it, and the amounts of absorbed and scattered light are equals when the size of particle reached $(80 \mathrm{~nm}$ ) (Khlebtsov NG 2008; Georgios D. Barmparis et al. 2015). AuNSps with (LSPR) in the visible range at approximately the $530 \mathrm{~nm}$ wavelength as demonstrated in Fig (1).

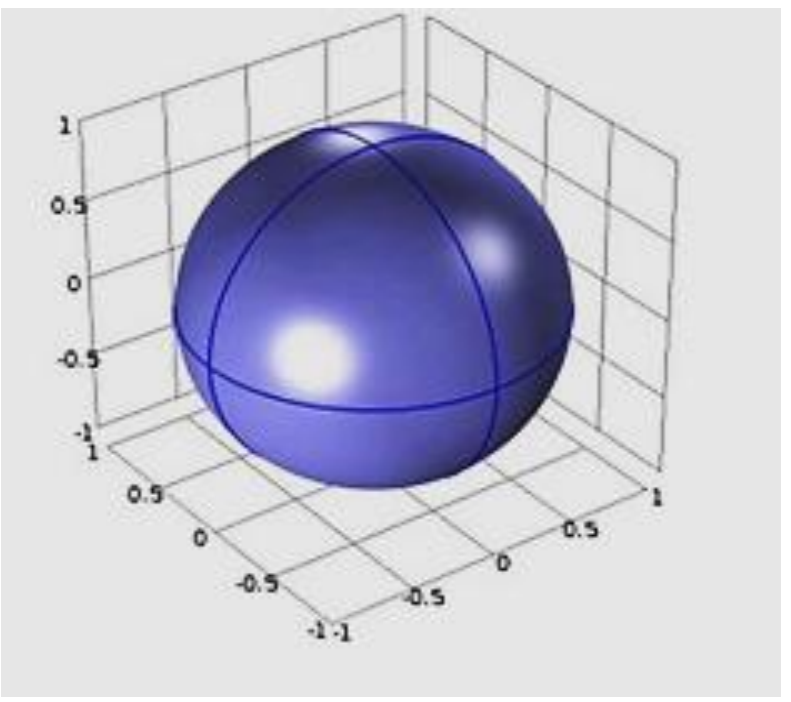

Fig 4: Representation of the nanosphere model.

1.1 Effect of increasing nanosphere size on scattering cross section

By rise the gold spherical nanostructure size, there is a significant red shift in the wavelength corresponding to the maximum efficiency of extinction. Furthermore, this guide can also be perceived in efficiency of scattering range as demonstrated in Fig (5). These results are identified with the reliance of free electron involvement in the dielectric function which can be adjusted by varying the volume of the structure (Megan A et al. 2013). As spherical nanostructures get bigger, the dipole reverberation tops red shifted. In any case, it is tracked down that rising the size of nanostructures brings about a rise in the scattering efficiency.

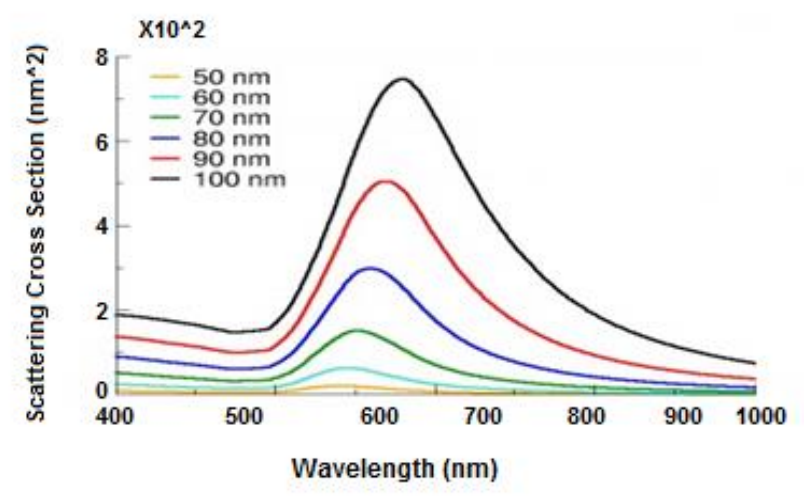

Fig 5: Scattering cross-section profile for a metallic nanosphere as function of wavelength for different diameters (Size).

\subsection{Environment effect on the scattering cross section}

The scattered light from the nanosphere will be increase when the refractive indices of the adjacent medium increased. Where there is an immediate connection between the refractive Indices of the adjacent medium and between the measurements of photons scattered from the molecule as demonstrated in Fig (6).

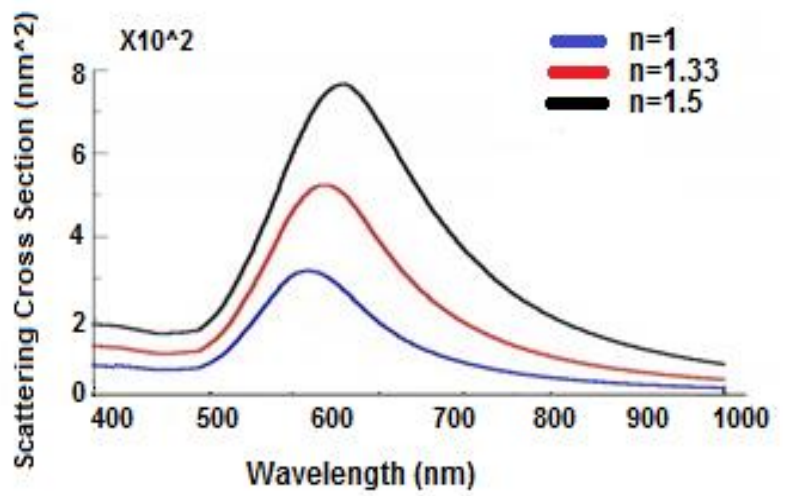

Fig 6: Effect of refractive indices for adjacent medium on scattering cross section of nanosphere.

1.3 Effect of gap distance between two nanospheres (nanosphere dimer)

For perpendicular (s-) polarized, for which the episode electrical fields are corresponding to the axis of dimer, the inter gap ( $\mathrm{g}$ ) between structures has considerable effects on the dimer's dominant dipolar plasmon resonance. As the nanostructures come near one another, the resonance peak is red shifted from the visible to (IR) range accompanying the gradual increase of scattering magnitude, as demonstrated in Fig (7). When the NPs are far away from one another, the resonance is close to the (LSPR) of a single particle, which is the situation of isolated single nanoparticle.

Distance dependent in nanostructure dimers have been investigated with (Alessia Polemi and Kevin L. Shuford (2012); Aning Ma et al. 2021). 


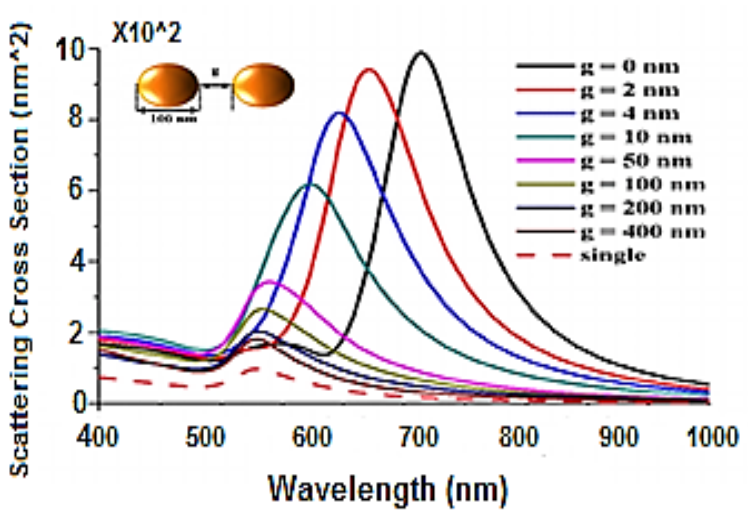

Fig 7: Effect of gap distance between two gold nanospheres (100nm) on scattering cross section (for s-polarization).

\section{2- Gold Nanorod}

A different example of anisotropic nanostructures is gold nanorod which is cylindrical shaped particles with hemispherical closures. Rather than AuNSps with (LSPR) in the noticeable reach at around the $530 \mathrm{~nm}$ wavelength as demonstrated in Fig (1), the longitudinal peak of (AuNRs) can be effectively tuning from (600 to $800 \mathrm{~nm}$ ) by the variety in their (length to width proportion) as demonstrated in Fig (2), this effect has been widely studied by (Babak Nikoobakht and Mostafa A. El-Sayed (2003); Mikhail Yu Tsvetkov et al. 2013). Specifically, great sensitive tune of the longitudinal resonance is clarified by the equation (13):

$\lambda_{\|}=\lambda_{\mathrm{p}} \sqrt{\varepsilon_{\mathrm{ib}}+\left(\frac{1}{\lambda_{\|}}-1\right)} \varepsilon_{\mathrm{m}}$

(LSPR) is red shifted as we increment the aspect ratio of (AuNRs), where $\left(\lambda_{\|}\right)$is the wavelength for longitudinal resonance; $\left(\lambda_{\mathrm{p}}\right)$ is the wavelength of the volume oscillations of an electron plasma; $\left(\varepsilon_{\mathrm{m}}\right)$ is the medium optical permittivity of; $\left(\varepsilon_{\mathrm{ib}}\right)$ is the contribution of inter band of electron transitions (Prashant K. Jain et al. 2006). It is known, that for AuNRs the absorption is the main contributor in the extinction spectra ( $\mathrm{Si}$ Yue Li and Min Wang (2012); Saumyakanti Khatua et al. 2014) that can be an issue for optical and medical imaging strategies.

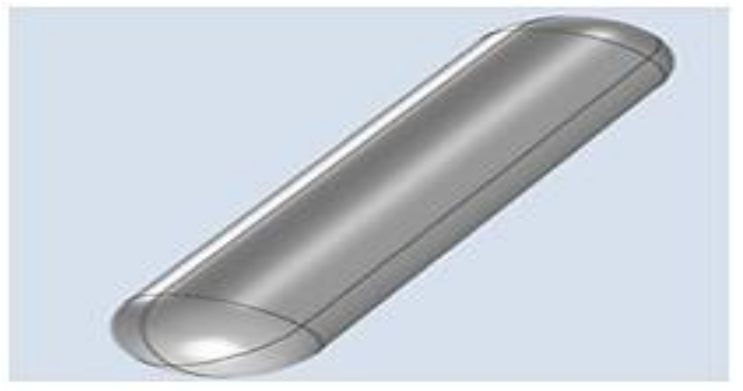

Fig 8: Representation of the nanorod model.
2.1 Effect of nanorod aspect ratio (length to width proportion)

One can see the red shift and strengthening of account of the scattering cross section when the aspect ratio gets higher as shown in Fig (9).

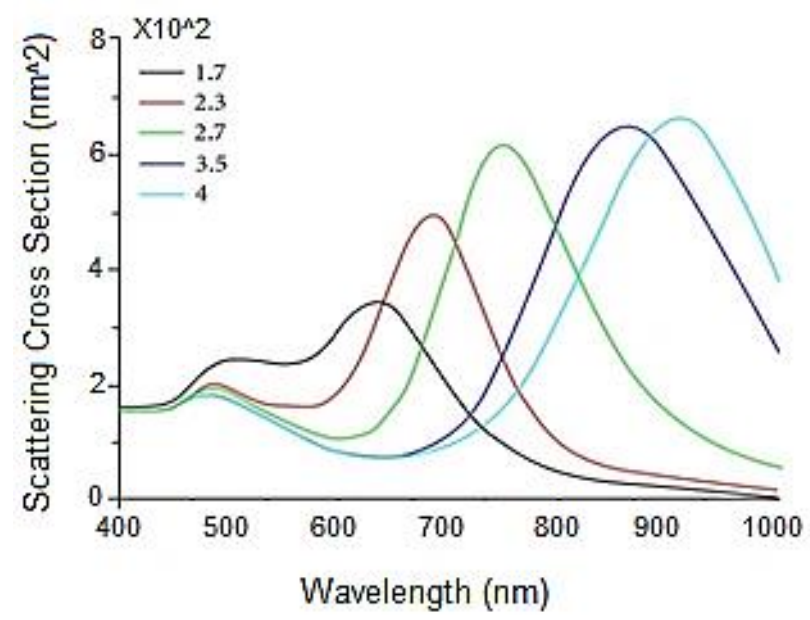

Fig 9: Effect of increasing the aspect ratio of nanorod.

\subsection{Effect of varying surrounding medium (refractive index)}

With varying the refractive indices of the adjacent medium, the scattering spectrum is red shifted and overlapped without any effect on amount of scattered light as shown in Fig (10).

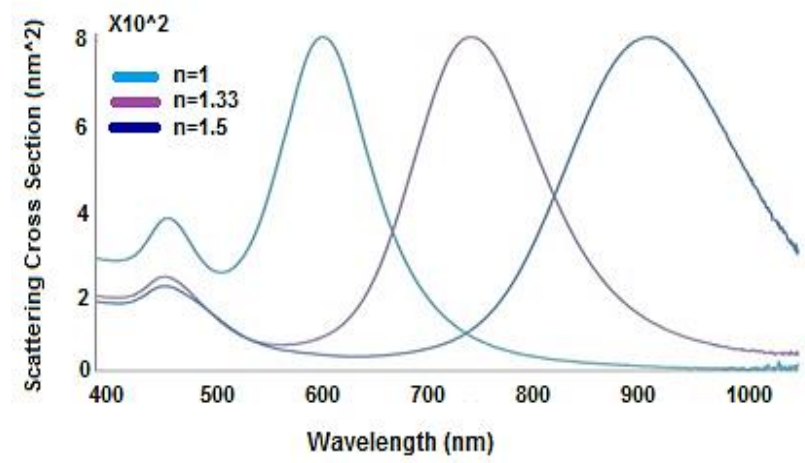

Fig 10: Effect of varying surrounding medium (refractive index).

\subsection{Effect of nanorod dimer on scattering cross section}

As the nanostructures approach one another, the resonance peak is red shifted from the visible to (IR) range accompanying the gradual increase in degree of scattering, as demonstrated in Fig (11). When the nanorods are distant from one another, the resonance is close to the (LSPR) of a single particle, which is the situation of lonely single nanoparticle. with increasing the gap distance between two nanorod the scattering cross section will decreasing and blue shifted as shown in Fig (11). 


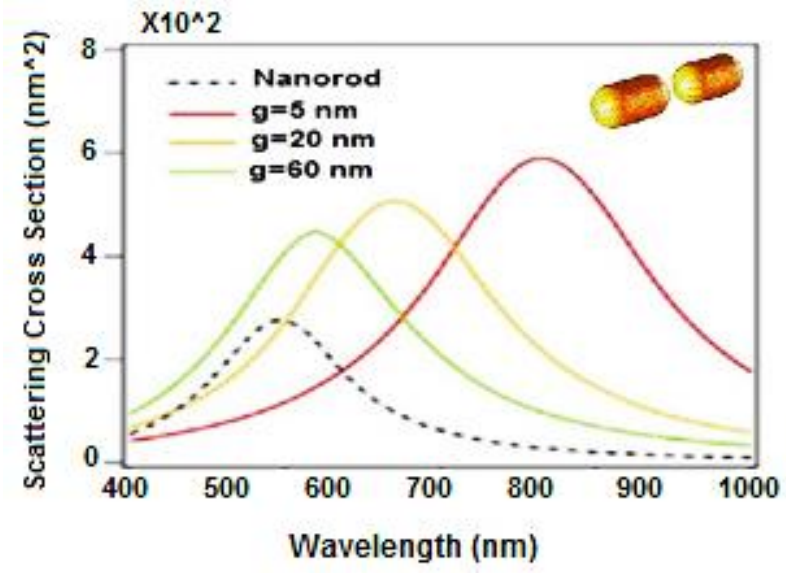

Fig 11: Effect of gap distance between nanorods on scattering cross section.

\section{3- Gold Nanostars}

As of late, branched and star formed nanostructures or gold nanostars (AuNSts) have pulled in a lot of significance in different medical applications because of the tunability of (LSPR) to the close to infrared (NIR) which is a diagnostic window. As demonstrated in Fig (3), and the presence of numerous sharp tips that can enormously improve occurrence electromagnetic fields (Feng Hao et al. 2007; P. Si et al. 2021). The superposition of the plasmons modes at core and the plasmons modes of the tips drastically rising the excitation of the bonding plasmons and results in huge enhancements in electrical field compared with those that would be initiated for singular tip (Chirico et al. 2015). This observable fact is also called antenna effect and is responsible for an increment in the extinction (absorption and scattering), however in the respective electrical field upgrade too (Chang Soo Kim et al. 2009). Because of large scattering, (AuNSts) are ideal contender to serve as contrast agent for doppler OCT and OCT imaging (Jung Heo et al. 2014; Vincenzo Amendola et al. 2017; Luca Minati et al. 2021).

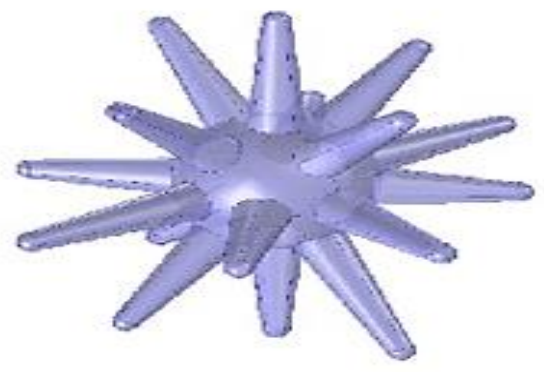

Fig 12: Representation of the nanostar model as defined by the analytical equation for its volume for a numeral of 18 spikes.

3.1 Effect of varying aspect ratio of nanostar (spike to core volume)

The frequency of electron reciprocates trembling in metallic nanostructures is controlled via the nanostructure's morphology and volume. Under the state of resonance, the free electron interaction between photons and metallic surface is strongest, and the greatest improvement in scattering and local light effect is formed on the surface of metallic structure. The spherical core of the AuNSts is set at radius $=30 \mathrm{~nm}$. The relation between the tips volume and the resonance peak of the AuNSts is calculated by varying the volume of the tip of the structure. while the tip long of (AuNSts) changing, the position of the SPR peak red shifted, the intensity of the resonance peak rises and the spectrum of resonance expand to some degree. The red shifts in the resonance peak are because of the size impact of nanostructure (Liu et al. 2015; Battulga Munkhbat et al. 2016; Ioannis G. Theodorou et al. 2018). As the tip turns out to be longer, potential photoinduced charge separation of the (AuNSts) along the polarization direction of the electrical fields increases, and therefore, the attraction between the negative and positive charges reduced. Accordingly, the number of times of oscillating electric dipole is increased, resonance frequency reduced and resonance peak will red shifted. Furthermore, the intensity of the resonance peak is owing to lighting rod effect turns out to be more clear as the tip turns out to be longer, and the charges stack at the tips with a higher concentration as demonstrated in Fig (13).

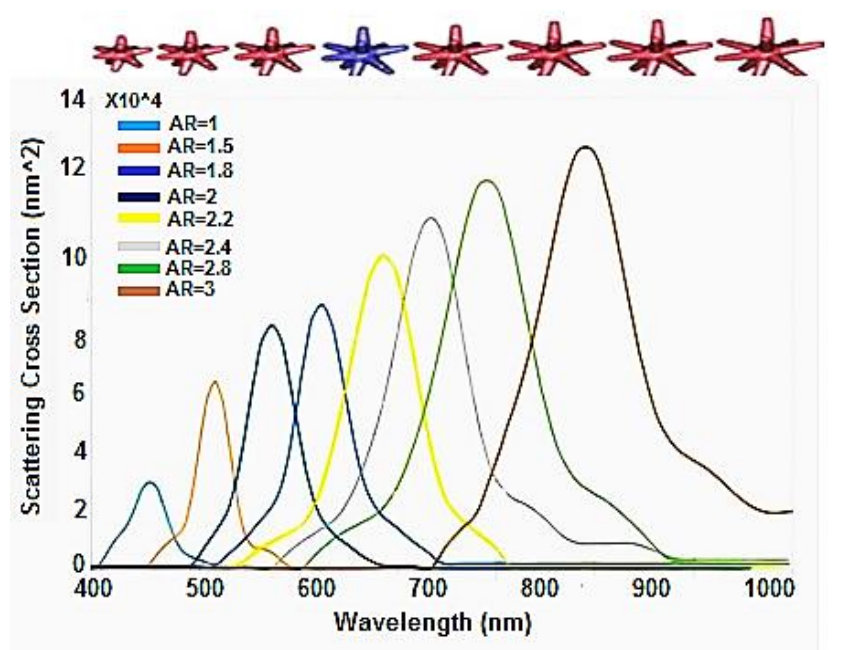

Fig 13: Effect of increasing spike size with fixed core volume $(\mathrm{R}=30 \mathrm{~nm})$ on nanostar scattering cross section.

\subsection{Effect of varying spikes number of nanostar}

When the number of spikes in branched nanostars increases, then sharp points will increase. At sharp points for the nanostar the electrons accumulate will be increase. It is more owing to the impact of incoming radiation on the nanostars. Nanostar plasmons begin to oscillate further plasma intensification occurs when nanostructure sharp points of the star increase due to those hot spots increase (Grace Lu et al. 2016; Andrew Fales et al. 2014; Wei Xiong et al. 2014). With increasing hot spots, the intensity of scattering in the surface plasmon increases and the peak of scattering the superficial red plasmon shifts as shown in Fig (14). 


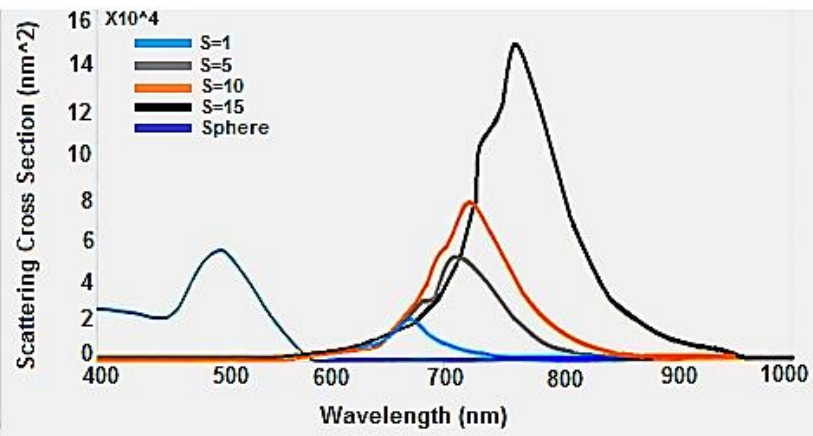

Fig 14: Effect of number of spikes of nanostar on scattering cross section.

\subsection{Effect of varying surrounding medium (refractive index)}

The resonance spectrum of (AuNSts) is extremely sensitive to the dielectric medium environs around them. The efficiency of scattering spectrum will be increase with increasing the refractive indices of adjacent medium and there are red shifts in spectrum will be seen as shown in Fig (15) (Ioannis H et al. 2016; Kyle D. Gilroy et al. 2016; Ted V. Tsoulos et al. 2018). The peak of scattering varied with dialectical environment of medium with $(n=1.5)$ is larger than that of medium with $(n=1.33)$ and $(n=1)$ because the dielectric coefficient of medium with $(n=1.5)$ is greater than that of medium with $(\mathrm{n}=1.33)$ and $(\mathrm{n}=1)$, according to the equation $\left(\omega(s)=\frac{\omega_{p}}{\sqrt{\varepsilon-1}}\right)$ As the dielectric coefficient increases, the resonant frequency decreases and the wavelength increases and the spectrum will red shifted.

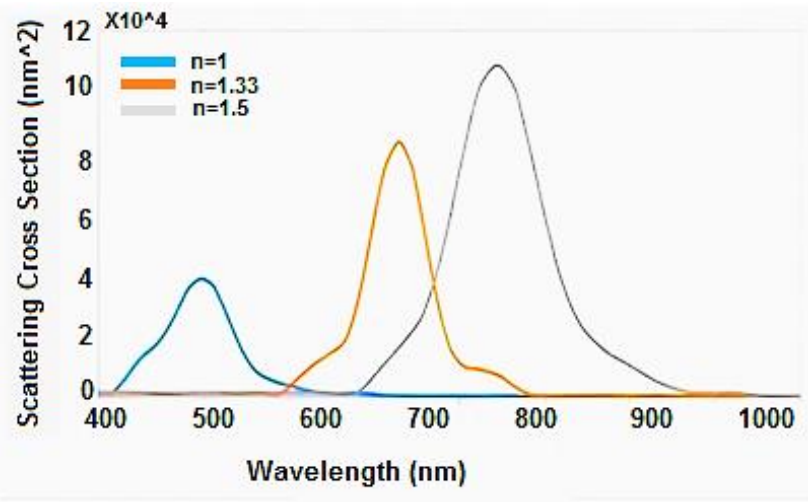

Fig 15: Effect of varying refractive index on scattering cross section of gold nanostar.

3.4 Effect of the gap between two nanostars dimer on scattering cross section

At the point when the two AuNSt are near one another, there is observable red shift in the peak of resonance with reduce the distance between particles, and the intensity of local field increases. As a result, by adjusting the AuNSt distance, the red shift in the near infrared band, and the intensity of local field can be efficiently increased by more than a hundredfold, which can be used in surface spectrum enhancement (T. V. Tsoulos et al. 2017; Stefan Harmsen et al. 2015). For the meantime, it can be seen that there are two peaks in resonance for the two AuNSts, in which the larger peak is due to the pairing effect between the AuNSts and the smaller peak is the inherent resonance peak of the AuNSt itself as demonstrated in Fig (16).

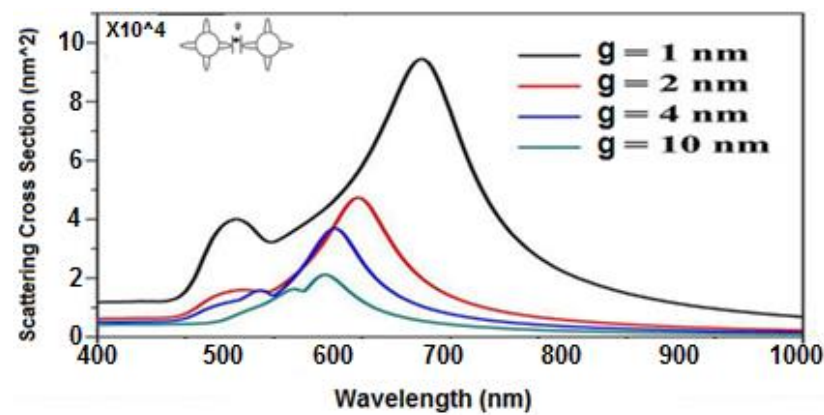

Fig 16: Effect of gap distance between two gold nanostars on scattering cross section.

\subsection{Influence of nanostars number}

As demonstrated in Fig (17) the spherical core in the center of the AuNSt has radius around (20) nm, the tip volume is (80) $\mathrm{nm}$ and gap distance around $(0.5) \mathrm{nm}$. The increment in the number of AuNSts will bring about the red shift of the peak of resonance, and the most extreme intensity of resonance. Larger peak is because of the pairing impact between the AuNSts, and the smaller peak is the inherent resonance peak of the AuNSt itself. The exceptionally improved electromagnetic fields created by pairing the plasmons between sharp tips and cores of two AuNSts in the wide conjunction area allocate the accommodation and specific detection of big molecules.

So, I propose utilizing gold nanostars dimer rather than single gold nanostars because of its improvement in light scattering thus it can to be gotten more clearly biomedical images.

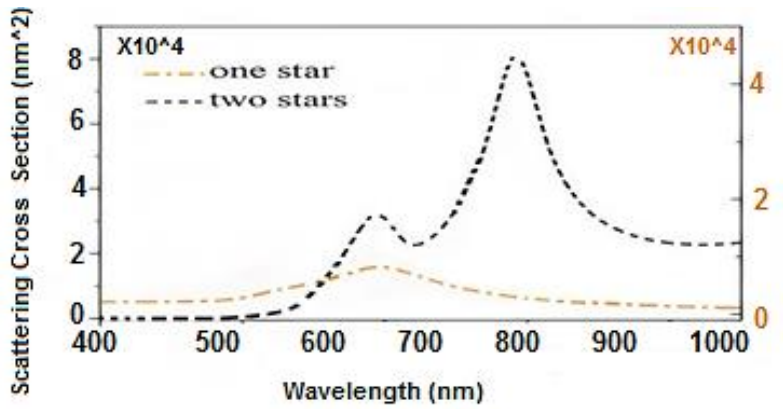

Fig 17: Effect of number of gold nanostars on normalized scattering cross section.

\section{CONCLUSIONS}

A significant trend in modern medicine is the substantial increase of the role of diagnostics in disease treatment strategies, including early-stage diagnostics and accurate detection of molecules in small concentrations. In view of above, the signal and response enhancement for various detection and imaging applications is a main challenge for modern diagnostics. Using of the localised plasmon resonance phenomena of plasmonic nanostructures is a future trend. 
Furthermore, the benefits of gold nanoparticles could be applied to increase the selectivity of therapy and diagnosis on the cell and tissue levels. Spherical gold nanoparticles are the simplest possible nanostructures with (SPR) in range (500 to $700 \mathrm{~nm}$ ) and the main contributor in the extinction cross section spectrum is Absorption, nanorods gold nanoparticles with (SPR) in range (600 to $800 \mathrm{~nm}$ ) and the main contributor in the extinction cross section spectrum is absorption, whereas at the moment of research related to the paper the benefits of plasmonic nanostars were clearly demonstrated in selected diagnostic applications as it has (SPR) in range (700 to 900 $\mathrm{nm}$ ) and the main contributor in the extinction cross section spectrum is scattering. In this paper nanostars were adopted both for optical imaging, spectroscopy analysis and for cell treatment due to (SPR) for it at tissue transparence window (700 to $1300 \mathrm{~nm}$ ). The plasmon resonant properties of nanostars were manipulated by modifying their size and surface properties cover to match localised plasmon resonance and the operational wavelength of the used equipment. The effectiveness of nanostars and gold nanoparticles was compared for selected bioimaging and sensing methods. Significant advantages of nanostars were highlighted in the paper. Due to the higher scattering cross-section in comparison to gold nanoparticles, large nanostars demonstrated better contrast properties for imaging applications for example confocal microscopy and optical coherence tomography. The broad peak and tunability of the nanostars localised plasmon resonance allows the effectiveness of laser optoporation to be maximised. Thus, the results of this study on the efficiency of nanostars and nanostars dimers have a broad scope of application and this broadens the potential range of utilisation for nanostars in nano biophotonics and biomedicine (Greenwood. Michael. 2021).

TABLE 1

COMPARISON BETWEEN MULTIPLE FACTORS AND ITS EFFECT ON SCATTERING CROSS SECTION FOR NANOSPHERES, NANOROD AND NANOSTARS.

\begin{tabular}{|c|c|c|c|}
\hline comparison factor & Nanosphere & Nanorod & Nanostar \\
\hline SPR Wavelength & 500 to $700 \mathrm{~nm}$ & 600 to $800 \mathrm{~nm}$ & 700 to $900 \mathrm{~nm}$ \\
\hline $\begin{array}{lr}\text { The } & \text { main } \\
\text { contributor in } & \text { in } \\
\text { extinction cross } & \text { section spectrum }\end{array}$ & Absorption & Absorption & Scattering \\
\hline $\begin{array}{l}\text { Effect of increasing } \\
\text { size on scattering } \\
\text { cross section }\end{array}$ & $\begin{array}{l}\text { Increasing } \\
\text { with red shift } \\
\text { in spectrum }\end{array}$ & $\begin{array}{lr}\text { Increasing with } \\
\text { red shift in } \\
\text { spectrum }\end{array}$ & $\begin{array}{l}\text { Increasing } \\
\text { with red shift } \\
\text { in spectrum }\end{array}$ \\
\hline $\begin{array}{lr}\text { Effect of } & \text { increase } \\
\text { refractive } & \text { indices } \\
\text { for } & \text { adjacent } \\
\text { medium } & \text { on } \\
\text { scattering } & \text { cross } \\
\text { section } & \\
\end{array}$ & $\begin{array}{l}\text { Increasing and } \\
\text { red shift }\end{array}$ & $\begin{array}{l}\text { red shifted and } \\
\text { overlapped } \\
\text { without any } \\
\text { effect on amount } \\
\text { of scattered light }\end{array}$ & $\begin{array}{l}\text { Increasing and } \\
\text { red shift }\end{array}$ \\
\hline $\begin{array}{l}\text { Effect of increasing } \\
\text { gap distance } \\
\text { between two } \\
\text { nanoparticles } \\
\text { (nanoparticle } \\
\text { dimer) }\end{array}$ & $\begin{array}{l}\text { Decrease in } \\
\text { scattering } \\
\text { cross section } \\
\text { spectrum and } \\
\text { blue shift }\end{array}$ & $\begin{array}{l}\text { Decrease in } \\
\text { scattering cross } \\
\text { section spectrum } \\
\text { and blue shift }\end{array}$ & $\begin{array}{l}\text { Decrease in } \\
\text { scattering } \\
\text { cross section } \\
\text { spectrum and } \\
\text { blue shift }\end{array}$ \\
\hline $\begin{array}{l}\text { Effect of increasing } \\
\text { Spikes number on } \\
\text { Scattering Cross } \\
\text { Section }\end{array}$ & & & $\begin{array}{l}\text { Increasing in } \\
\text { scattering } \\
\text { cross section } \\
\text { spectrum and } \\
\text { red shift }\end{array}$ \\
\hline
\end{tabular}

\section{Authors Contribution}

1. Conception or design of the work: by Eng. Kholoud Ramadan Abdelhady and Associate Prof. Bedir B.Yousif.

2. Data collection and tools: by Eng. Kholoud Ramadan Abdelhady.

3. Data analysis and interpretation: by Eng. Kholoud Ramadan Abdelhady.

4. Funding acquisition: I don't receive any financial support or the research, authorship and/ or publication of my article.

5. Investigation: by Prof. Hossam El- Din Moustafa and Associate Prof. Bedir B.Yousif

6. Methodology: by Associate Prof. Bedir B.Yousif.

7. Project administration: by Eng. Kholoud Ramadan Abdelhady.

8. Resources: by Eng. Kholoud Ramadan Abdelhady and Associate Prof. Bedir B.Yousif.

9. Software: by Eng. Kholoud Ramadan Abdelhady -using RF Module of (COMSOL Multiphysics 5.0)

10. Supervision The work was supervised: by Prof. Hossam El- Din Moustafa and Associate Prof. Bedir B.Yousif.

11. Drafting the article: by Eng. Kholoud Ramadan Abdelhady.

12. Critical revision of the article: by Prof. Hossam El- Din Moustafa and Associate Prof. Bedir B.Yousif.

\section{FUNDING STATEMENT:}

The author did not receive any financial support of the research authorship and publication of this article.

\section{DECLARATION OF CONFLICTING INTERESTS STATEMENT:}

The author declared that there are no potential conflicts of interest with respect to the research authorship or publication of this article.

\section{REFERENCES}

[1] Kim EY, Kumar D, Khang G \& Lim D-K.: Recent advances in gold nanoparticlebased bioengineering applications. J Mater Chem B 3(43): 8433-8444. (2015) https://doi.org/10.1039/c5tb01292a.

[2] Kanika Khurana, Neena Jaggi. Localized Surface Plasmonic Properties of Au and Ag Nanoparticles for Sensors: a Review. Plasmonics 2021, 16 (4), 981-999. https://doi.org/10.1007/s1 1468-021-01381-1

[3] Luca Guerrini, Eduardo Garcia-Rico, Ana O'Loghlen, Vincenzo Giannini, Ramon Alvarez-Puebla. Surface-Enhanced Raman Scattering (SERS) Spectroscopy for Sensing and Characterization of Exosomes in Cancer Diagnosis. Cancers 2021, $13 \quad$ (9), 2179. https://doi.org/10.3390/cancers13092179

[4] Versiani AF, Andrade LM, Martins EMN, Scalzo S, Geraldo JM, Chaves CR, Ferreira DC, Ladeira M, Guatimosim S, Ladeira LO and da Fonseca FG.: Gold nanoparticles and their applications in biomedicine. Fut Virol 11(4): 293-309. (2016) https://doi.org/10.2217/fvl-2015-0010.

[5] Sousa-Castillo, A.; Comesaña-Hermo, M.; Rodríguez-González, B.; Pérez-Lorenzo, M.; Wang, Z.; Kong, X.-T.; Govorov, A. O.; CorreaDuarte, M. A.: Boosting Hot Electron-Driven Photocatalysis through Anisotropic Plasmonic Nanoparticles with Hot Spots in $\mathrm{Au}-\mathrm{TiO} 2$ Nanoarchitectures. J. Phys. Chem. C 120 (21): 11690-11699. (2016) https://doi.org/10.1021/acs.jpcc.6b02370.

[6] Bhamidipati, M.; Fabris, L.: Multiparametric Assessment of Gold Nanoparticle Cytotoxicity in Cancerous and Healthy Cells: The Role of Size, Shape, and Surface Chemistry. Bioconjugate Chem 28 (2): 449460. (2017) https://doi.org/10.1021/acs.bioconjchem.6b00605.

[7] Duke University. (2020, July 21). Silver-plated gold nanostars detect early cancer biomarkers: New optical sensing platform can detect 
genomic cancer biomarkers directly in patient tissues. ScienceDaily. Retrieved December 4, 2021 from www.sciencedaily.com/releases/2020/07/200721132731.htm.

[8] Jana D, Matti C, He J and Sagle L.: Capping agent-free gold nanostars show greatly increased versatility and sensitivity for biosensing. Anal Chem 87(7): 3964-3972. https://doi.org/10.1021/acs.analchem.5b00014.

[9] Vendrell M, Maiti KK, Dhaliwal K, et al.: Surface-enhanced Raman scattering in cancer detection and imaging. Trends Biotechnol 31(4): 4957. (2013) https://doi.org/10.1016/j.tibtech.2013.01.013.

[10] Harmsen S, Huang R, Wall MA, et al.: Surface-enhanced resonance Raman scattering nanostars for high-precision cancer imaging. Sci Transl Med 7(271): pp. 271ra7. (2015) https://doi.org/10.1126/scitranslmed.3010633.

[11] Hsiangkuo, Y.; Christopher, G. K.; Hanjun, H.; Christy, M. W.; Gerald, A. G.; Tuan, V.-D.: Gold nanostars: surfactant-free synthesis, 3D modelling, and two-photon photoluminescence imaging. Nanotechnology $23 \quad$ (7). (2012) https://doi.org/10.1088/09574484/23/7/075102.

[12] Zhihua, X.; Kong, W.; Cheng, L.; Ma, A.; Lu, X.; Wang, Y.; Zhang, X.; Ya Zhang, X.: Universal Three-Dimensional Polarization-Dependent Optical Properties in Anisotropic Plasmonic Nanostar: a Route Boosting Single Particle 3D Orientation Determination and Orientation-Unlimited Polarization Information Detection. Plasmonics 10(1). (2015) https://doi.org/10.1007/s11468-015-9910-9.

[13] Hale, G. M.; Querry, M. R.: Optical Constants of Water in the 200-nm to 200- $\mu \mathrm{m}$ Wavelength Region. Appl. Opt. 12 (3): 555-563. (1973) https://doi.org/10.1364/AO.12.000555.

[14] Johnson, P. B.; Christy, R. W.: Optical Constants of the Noble Metals. Phys. Rev. B 6(12): 4370-4379. (1972) https://doi.org/10.1103/PhysRevB.6.4370.

[15] Haes AJ, Van Duyne RP.: A nanoscale optical blosen-sor: sensitivity and selectivity of an approach based onthe localized surface plasmon resonance spectroscopyof triangular silver nanoparticles.JAmChemSoc, 124(35): 10596 - 10604. (2002) https://doi.org/10.1021/ja020393x.

[16] Ginting, R. T.; Kaur, S.; Lim, D.-K.; Kim, J.-M.; Lee, J. H.; Lee, S. H.; Kang, J.-W.: Plasmonic Effect of Gold Nanostars in Highly Efficient Organic and Perovskite Solar Cells. ACS Appl. Mater. Interfaces. 9 (41): 36111-36118. (2017) https://doi.org/10.1021/acsami.7b11084.

[17] Anker JN, Hall WP, Lyandres O, Shah NC, Zhao J,Van Duyne RP.: Biosensing with plasmonic nano-sensors.Nat Mater. 7(6): 442 - 453. (2008) https://doi.org/10.1142/9789814287005_0032.

[18] Jain PK, Lee KS, El-Sayed IH, El-Sayed M.: Calculated absorption and scattering properties of gold nanoparticles of different size, shape, and composition: applications in biological imaging and biomedicine. J Phys Chem B. 110(14): 7238-7248. (2006) https://doi.org/10.1021/jp057170o.

[19] Xianghua Meng, Aravind Baride, and Chaoyang Jiang. Ligand Controlled Morphology Evolution of Active Intermediates for the Syntheses of Gold Nanostars. Langmuir. 32(26): 6674-6681. (2016) https://doi.org/10.1021/acs.langmuir.6b01592.

[20] A. Swarnapali D. S. Indrasekara, Roney Thomas and Laura Fabris.: Plasmonic properties of regiospecific core-satellite assemblies of gold nanostars and nanospheres. PCCP. 17 (33): 21133-21142. (2015) https://doi.org/10.1039/C4CP04517C.

[21] Helena de Puig, Justina O. Tam, Chun-Wan Yen, Lee Gehrke, and Kimberly Hamad-Schifferli.: Extinction Coefficient of Gold Nanostars. $\begin{array}{llllll}\text { J. Phys. Chem. } & \text { C. } 119 & \text { (30), 17408-17415. (2015) }\end{array}$ https://doi.org/10.1021/acs.jpcc.5b03624.

[22] Khlebtsov NG: Optics and biophotonics of nanoparticles with a Plasmon resonance. Quantum Electron. 38(6): 504-529. (2008) https://doi.org/10.1070/QE2008v038n06ABEH013829.

[23] Georgios D. Barmparis, Zbigniew Lodziana, Nuria Lopez and Ioannis N. Remediakis.: Nanoparticle shapes by using Wulff constructions and first-principles calculations. Beilstein J. Nanotechnol. 6 (1): 361-368. (2015) https://doi.org/10.3762/bjnano.6.35.

[24] Megan A. Mackey, Farhat Saira, Mahmoud A. Mahmoud, and Mostafa A. El-Sayed.: Inducing Cancer Cell Death by Targeting Its Nucleus: Solid Gold Nanospheres versus Hollow Gold Nanocages. Bioconjugate Chem. 24(6): 897-906. (2013) https://doi.org/10.1021/bc300592d.

[25] Alessia Polemi and Kevin L. Shuford.: Distance dependent quenching effect in nanoparticle dimmers. J. Chem. Phys. 136(18): 184703. (2012) https://doi.org/10.1063/1.4711759.

[26] Aning Ma, Wenjing Wei, Guojian Li, Yurong Wang, Di Geng, Zhongqiang Zhang. Insight into excitation wavelength and hotspot effect of noble metal nanoparticles dimer. Optoelectronics Letters 2021, 17 (10), 577-580. https://doi.org/10.1007/s11801-021-0200-9

[27] Babak Nikoobakht and Mostafa A. El-Sayed.: Preparation and growth mechanism of gold nanorods (NRs) using seed-mediated growth method. Chem Mater 15(10): 1957-1962. (2003) https://doi.org/10.1021/cm0207321.

[28] Mikhail Yu Tsvetkov, Boris N Khlebtsov, Vitaly A Khanadeev, Victor N Bagratashvili, Peter S Timashev, Mikhail I Samoylovich and Nikolai G Khlebtsov.: SERS substrates formed by gold nanorods deposited on colloidal silica films. Nanoscale research letters. 8(1): 250 (2013) https://doi.org/10.1186/1556-276X-8-250.

[29] Prashant K. Jain, Kyeong Seok Lee, Ivan H. El-Sayed, and Mostafa A. El-Sayed.: Calculated absorption and scattering properties of gold nanoparticles of different size, shape, and composition: applications in biological imaging and biomedicine. J Phys Chem B 110(14): 72387248. (2006) https://doi.org/10.1021/jp057170o.

[30] Si Yue Li and Min Wang.: Branched metal nanoparticles: a review on wet-chemical synthesis and biomedical applications. Nano Life 2(1): 1230002. (2012) https://doi.org/10.1142/S1793984411000311.

[31] Saumyakanti Khatua, Pedro M. R. Paulo, Haifeng Yuan, Ankur Gupta, Peter Zijlstra, and Michel Orrit.: Resonant Plasmonic Enhancement of Single-Molecule Fluorescence by Individual Gold Nanorods. ACS Nano 8(5): 4440-4449 (2014). https://doi.org/10.1021/nn406434y.

[32] Feng Hao, Colleen L. Nehl, Jason H. Hafner, and Peter Nordlander.: Plasmon resonances of a gold nanostar. Nano Lett 7(3): 729-732. (2007) https://doi.org/10.1021/n1062969c.

[33] P. Si, N. Razmi, O. Nur, S. Solanki, C. M. Pandey, R. K. Gupta, B. D. Malhotra, M. Willander and A. de la Zerda. Gold nanomaterials for optical biosensing and bioimaging Nanoscale Adv.2021 (3): 2679-2698. (2021) https://doi.org/10.1039/D0NA00961J.

[34] Chirico, Giuseppe, Borzenkov, Mykola, Pallavicini, Piersandro.: Gold nanostars synthesis, properties and biomedical application. SPRingerBriefs in Materials. (2015).

[35] Chang Soo Kim, Lih-Huei L. Liaw, Petra Wilder-Smith, Yeh-Chan Ahn , and Zhongping Chen. : Enhanced detection of early-stage oral cancer in vivo by optical coherence tomography using multimodal delivery of gold nanoparticles. J Biomed Opt 14 (3): 034008. (2009) https://doi.org/10.1117/1.3130323.

[36] Jung Heo, Eunji Jang, Seungjoo Haam, Seung Jae Oh, Yong-Min Huh, Jin-Suck Suh, Euiheon Chung, and Chulmin Joo. In vivo photothermal optical coherence tomography of targeted mouse brain tumors using gold nanostars. Proc Lasers and Electro-Optics (CLEO) OSA: 1-2. (2014) https://doi.org/10.1364/CLEO_SI.2014.SM4P.3.

[37] Vincenzo Amendola, Roberto Pilot, Marco Frasconi, Onofrio M Maragò and Maria Antonia Iatì.: Surface Plasmon resonance in gold nanoparticles: a review. J Phys: Condens Matter 29(20): 203002. (2017) https://doi.org/10.1088/1361-648X/aa60f3.

[38] Luca Minati, Devid Maniglio, Filippo Benetti, Andrea Chiappini and Giorgio Speranza. Multimodal Gold Nanostars as SERS Tags for OpticallyDriven Doxorubicin Release Study in Cancer Cells. Materials 14 (23): pp. 7272. (2021) https://doi.org/10.3390/ma14237272.

[39] Liu, Yang, Yuan, Hsiangkuo, Fales, Andrew M, Register, Janna K, VoDinh, Tuan.: Multifunctional gold nanostars for molecular imaging and cancer therapy. Front. Chem. 3 (51): (2015) https://doi.org/10.3389/fchem.2015.00051.

[40] Battulga Munkhbat, Johannes Ziegler, Hannes Pöhl, Christian Wörister, Dmitry Sivun, Markus C. Scharber, Thomas A. Klar, and Calin Hrelescu.: Hybrid Multilayered Plasmonic Nanostars for Coherent Random Lasing. J. Phys. Chem. C. 120(41): 23707-23715. (2016) https://doi.org/10.1021/acs.jpcc.6b05737.

[41] Ioannis G. Theodorou, Qianfan Jiang, Lukas Malms, Xiangyu Xie, R. Charles Coombes, Eric O. Aboagye, Alexandra E. Porter, Mary P. Ryan and Fang Xie. Fluorescence enhancement from single gold nanostars: towards ultra-bright emission in the first and second near-infrared biological windows. Nanoscale. 10 (33): 15854-15864. (2018) https://doi.org/10.1039/C8NR04567D.

[42] Grace Lu, Tori Z. Forbes and Amanda J. Haes. SERS detection of uranyl using functionalized gold nanostars promoted by nanoparticle shape and size. The Analyst. 141 (17): 5137-5143. (2016) https://doi.org/10.1039/c6an00891g.

[43] Andrew Fales, Hsiangkuo Yuan and Tuan Vo-Dinh.: Development of hybrid silver-coated gold nanostars for nonaggregated surface-enhanced Raman scattering. The Journal of Physical Chemistry C. 118(7): 37083715. (2014) https://pubs.acs.org/doi/10.1021/jp4091393. 
[44] Wei Xiong, Romiza Mazid, Lim Wei Yap, Xinyong Li and Wenlong Cheng: Plasmonic Caged Gold Nanorods for Near-infrared Light Controlled Drug Delivery. Nanoscale 6(23): 14388-14393. (2014) https://doi.org/10.1039/C4NR04400B.

[45] Ioannis H. Karampelas, Kai Liu, Fatema Alali, and Edward P. Furlani.: Plasmonic Nanoframes for Photothermal Energy Conversion. J. Phys. Chem. C. 120(13): 7256-7264. (2016) https://doi.org/10.1021/acs.jpcc.5b12743.

[46] Kyle D. Gilroy, Aleksey Ruditskiy, Hsin-Chieh Peng, Dong Qin, and Younan Xia. : Bimetallic Nanocrystals: Syntheses, Properties, and Applications. Chem. Rev. 116(18): 10414-10472. (2016) https://doi.org/10.1021/acs.chemrev.6b00211.

[47] Ted V. Tsoulos, Supriya Atta ,Maureen J. Lagos , Philip E. Batson ,George Tsilomelekis , Laura Fabris .Rational Design of Gold Nanostars with Tailorable Plasmonic Properties. (2018) https://doi.org/10.26434/chemrxiv.6552743.v1.

[48] T. V. Tsoulos, L. Han, J. Weir, H. L. Xin and L. Fabris.: A closer look at the physical and optical properties of gold nanostars: an experimental and computational study. Nanoscale. 9(11): 3766-3773. (2017) https://doi.org/10.1039/C6NR09091E.

[49] Stefan Harmsen, Ruimin Huang, Matthew A. Wall, Hazem Karabeber, Jason M. Samii, Massimiliano Spaliviero, Julie R. White, Sébastien Monette, Rachael O'Connor, Kenneth L. Pitter, Stephen A. Sastra, Michael Saborowski, Eric C. Holland, Samuel Singer, Kenneth P. Olive, Scott W. Lowe, Ronald G. Blasberg and Moritz F. Kircher: Surfaceenhanced resonance Raman scattering nanostars for high-precision cancer imaging. Sci. Transl. Med. 7 (271): 271ra7. (2015) https://doi.org/10.1126/scitranslmed.3010633.

[50] Greenwood. Michael. (2018, October 08). Nanostar Applications in Biomedicine. News-Medical. Retrieved on December 04, 2021 from https://www.news-medical.net/life-sciences/Nanostar-Applications-inBiomedicine.aspx.
Arabic Title

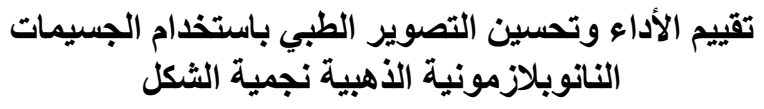

\section{Abstract in Arabic:}

تقدم هذه الورقة البحثية نظرة ثاقبة حول فحص خصائص الجسيمات النانويلازمونية

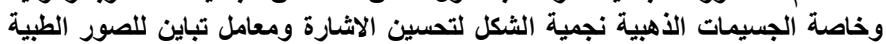

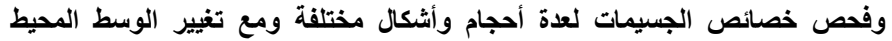

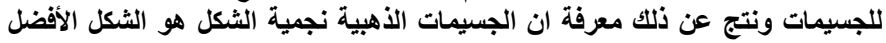

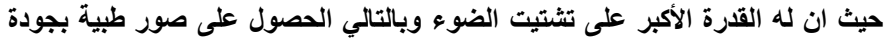

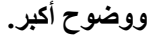

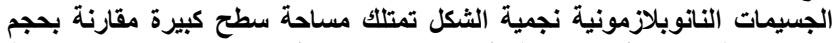

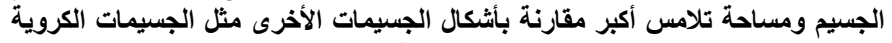

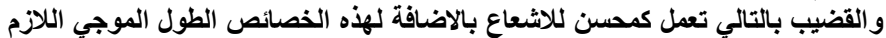

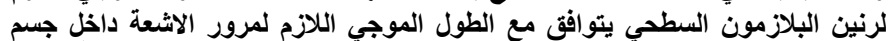

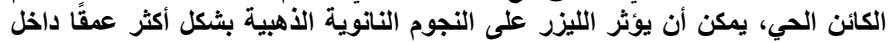

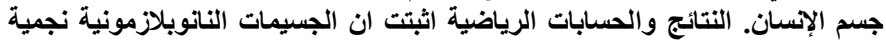

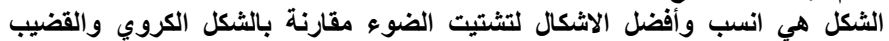

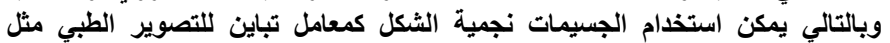
الفحص المجهري متحد البؤز والتصوير المقطعي البصري. 